\title{
Far-lateral transcondylar approach: surgical technique and its application in neurenteric cysts of the cervicomedullary junction
}

\author{
Report of two cases
}

\author{
James K. Liu, M.D., And William T. Couldwell, M.D., Ph.D. \\ University of Utah School of Medicine, Salt Lake City, Utah
}

\begin{abstract}
Neurenteric cysts are rare benign lesions of the central nervous system that are lined by endodermal cell-derived epithelium. Although they occur mostly in the spine, they can occur intracranially, most often in the posterior fossa. Neurenteric cysts that are located in the anterior cervicomedullary junction are even rarer and often require a skull base approach for adequate resection. The authors describe two cases of neurenteric cysts arising from the cervicomedullary junction that were resected via a far-lateral transcondylar approach. They discuss the surgical approach and operative nuances involved in removing these lesions, and review the clinical presentation of neurenteric cysts in this region as well as the neuroimaging characteristics, histopathological findings, and surgical management. Intraoperative videos are presented.
\end{abstract}

\section{KEY WORDS • neurenteric cyst • enterogenous cyst • cervicomedullary junction • far-lateral transcondylar approach}

Neurenteric cysts, also known as enterogenous, endodermal, neuroenteric, respiratory, or bronchogenic cysts, are rare benign lesions of the central nervous system that are lined by endodermal cell-derived epithelium. ${ }^{6,13,26}$ They are most frequently found in the intradural extramedullary space in the lower cervical and upper thoracic spine., 30,22 Intracranial neurenteric cysts are even rarer, with the majority located in the posterior fossa. ${ }^{6,8,13,14}$ Spinal neurenteric cysts are often connected by a fibrous tract, fistula, or cleft to structures derived from the primitive gut in the thoracic or abdominal cavities and are commonly associated with vertebral anomalies such as vertebral body dysgenesis, split spinal cord malformations, hemivertebra, segmentation abnormalities, and spina bifida. ${ }^{7,2,22,24}$ This is not the case with intracranial neurenteric cysts. ${ }^{6}$ Although the exact pathogenesis remains unknown, intracranial neurenteric cysts are thought to arise from a failure of dissolution of the transient neurenteric canal between the foregut or the respiratory buds and the notochord during notochordal development. ${ }^{8,14,17}$

Posterior fossa neurenteric cysts constitute more than $90 \%$ of the intracranial form of these cysts, and they occur mostly along the midline. In a review by Bejjani, et al., ${ }^{6}$ the

Abbreviations used in this paper: $\mathrm{CPA}=$ cerebellopontine angle; FLAIR = fluid-attenuated inversion-recovery; $\mathrm{MR}=$ magnetic resonance; $\mathrm{VA}=$ vertebral artery. most common locations of posterior fossa neurenteric cysts were anterior to the brainstem (51\%) or within the fourth ventricle $(21 \%)$. Also, $18 \%$ of the cysts extended into the cervical canal and $17 \%$ were located primarily in the CPA. Neurenteric cysts that are located in the anterior cervicomedullary junction are even rarer, and often require a skull base approach for adequate resection. ${ }^{1,12,14,16,20,21} \mathrm{We}$ describe two cases of neurenteric cysts arising from the cervicomedullary junction that were resected via a far-lateral transcondylar approach. We discuss the surgical approach and operative nuances involved in removing these lesions, and we review the clinical presentation of neurenteric cysts in this region as well as the neuroimaging characteristics, histopathological findings, and surgical management.

\section{CASE REPORTS}

\section{Case 1}

History and Examination. This 46-year-old woman presented with pain in the right shoulder, right ear, and interscapular area. Results of the neurological examination were normal, with the exception of a markedly diminished gag reflex on the right side. A laryngoscopic examination performed in the otolaryngology service demonstrated normal vocal cord movement. 
Neuroimaging. Admission MR imaging demonstrated a lesion in the cervicomedullary cistern involving the ninth and 10th cranial nerves on the right side (Fig. 1). The lesion was isointense on $T_{1}$-weighted and hyperintense on $T_{2}-$ weighted and FLAIR images. No enhancement was observed after $\mathrm{Gd}$ administration.

Operation. The patient underwent a far-lateral transcondylar approach (described in detail later) for gross-total resection of the lesion. Intraoperatively, a white cystic lesion was identified beneath the flocculus, located deep to the ninth and 10th cranial nerve complex (Fig. 1, Video 1). The structure appeared gelatinous and was covered by a thin, translucent membrane. The lesion was dissected from the lower cranial nerves and removed entirely.

Video 1. Intraoperative video of the patient in Case 1. A right far-lateral transcondylar approach was performed for resection of a neurenteric cyst in the cervicomedullary junction. (Click here to view with Windows Media Player, orhere to view with RealPlayer.)

Histopathological Examination. Grossly, the specimen appeared to be a white, translucent mucoid substance. Histological examination revealed a cyst wall lined by ciliated columnar epithelium (Fig. 2) that yielded positive results on staining for keratin markers, suggesting an endodermal origin. The final diagnosis was neurenteric cyst.

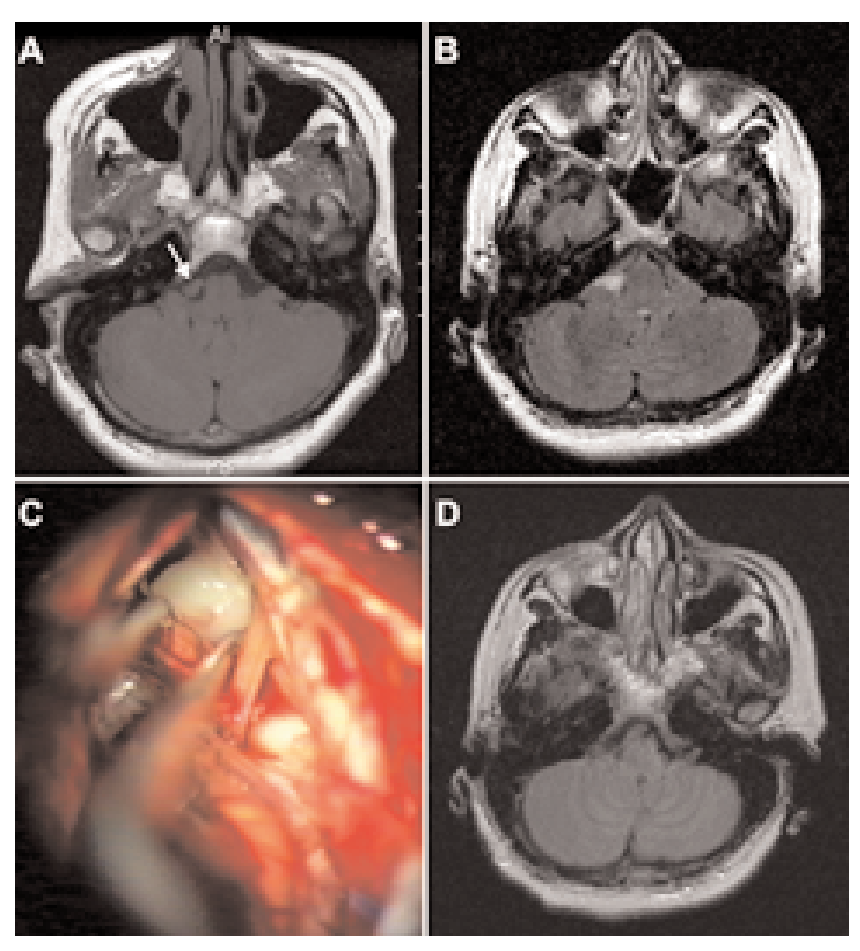

Fig. 1. Case 1. A and B: Preoperative MR images $\left(A, T_{1^{-}}\right.$ weighted axial image; B, FLAIR axial image) demonstrating a neurenteric cyst in the cervicomedullary cistern (arrow) involving the ninth and 10th cranial nerves on the right side. Note that the cyst is isointense on the $\mathrm{T}_{1}$-weighted image and hyperintense on the FLAIR image. There was no enhancement after Gd administration. $\mathrm{C}$ : Intraoperative photograph depicting a white cystic lesion next to the ninth and 10th cranial nerve complex. D: Postoperative MR image (FLAIR axial image) demonstrating gross-total resection of the neurenteric cyst.

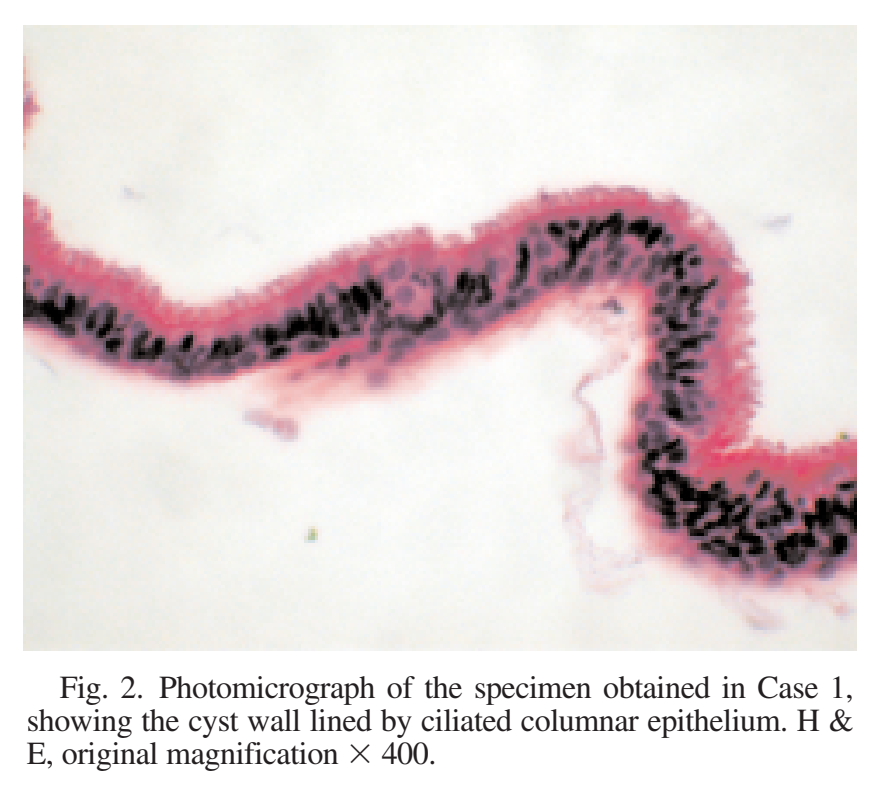

Postoperative Course. The patient did well postoperatively and had an uneventful course. She had no difficulty with swallowing, and sensation in her right posterior pharynx improved after surgery. Postoperative MR imaging demonstrated a complete resection of the lesion (Fig. 1).

Case 2

History and Examination. This 26-year-old woman presented with headaches. Results of the neurological examination were normal.

Neuroimaging. Admission MR imaging demonstrated a smoothly marginated mass occupying the right cervicomedullary junction and extending anterior to the brainstem, with mild mass effect (Fig. 3). It was hyperintense on $\mathrm{T}_{1}$ weighted images, slightly hyperintense on $\mathrm{T}_{2}$-weighted images, and minimally enhanced after $\mathrm{Gd}$ administration.

Operation. This lesion had been followed with serial neuroimaging, and showed some growth after a 1-year interval. Because of the size of the cyst and the fact that it was already showing some mass effect on the brainstem, the patient underwent a far-lateral transcondylar approach (described in detail later) for gross-total resection of the lesion. Intraoperatively, a yellow-white, custardlike cyst was identified surrounding and enveloping the ninth and 10th cranial nerves (Fig. 3, Video 2). The inferior aspect of the cyst was mobilized from the 12th cranial nerve and the cyst was opened to remove some of the colloid material to decompress the lesion. The remaining cyst wall was dissected off the anterior aspect of the brainstem and the VA up to the vertebrobasilar junction. All of the cranial nerves were left intact.

Video 2. Intraoperative video of the patient in Case 2. A right far-lateral transcondylar approach was performed for resection of a neurenteric cyst in the cervicomedullary junction. Note the vertebrobasilar junction and the lower cranial nerves that are preserved at the end of the operation. (Clickhere to view with Windows Media Player, on here to view with RealPlayer.) 

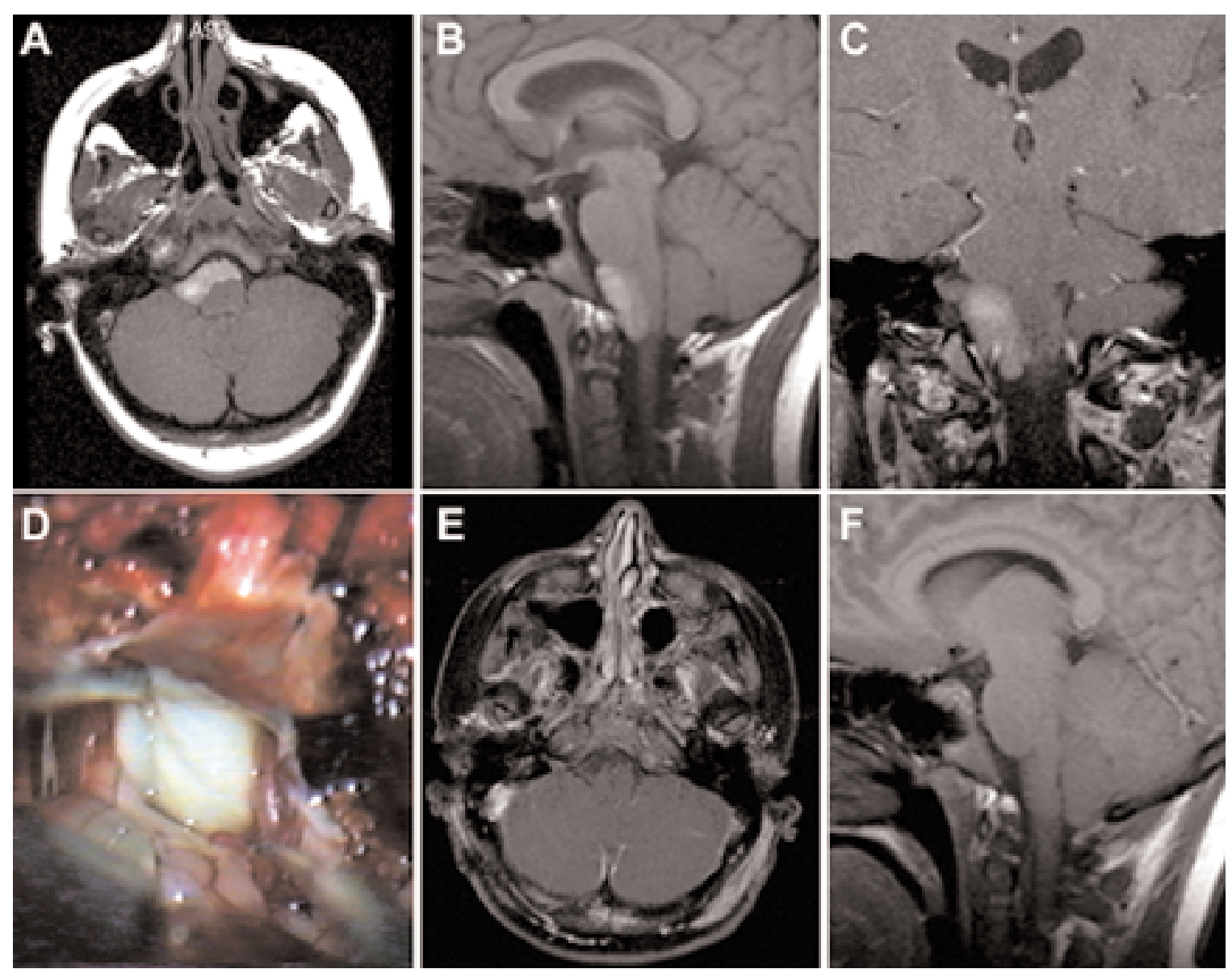

Fig. 3. Case 2. A-C: Preoperative MR images (A, $T_{1}$-weighted axial image; $B, T_{1}$-weighted sagittal image; $C$, post-Gd $T_{1}$-weighted coronal image) demonstrating a neurenteric cyst occupying the right cervicomedullary junction and extending anterior to the brainstem with mass effect. In this case, the cyst is hyperintense on $\mathrm{T}_{1}$-weighted images and enhances after Gd administration. D: Intraoperative photograph depicting a white cystic lesion surrounding and enveloping the ninth and 10th cranial nerves. E and F: Postoperative MR images (E, post-Gd $\mathrm{T}_{1}$-weighted axial image; $\mathrm{F}, \mathrm{T}_{1}$-weighted sagittal image) demonstrating gross-total resection of the neurenteric cyst.

Histopathological Examination. Histological examination revealed a cyst wall lined with ciliated simple cuboidal-tocolumnar epithelium (Fig. 4). The cyst contents consisted of anucleated, keratinous debris. The final diagnosis was a neurenteric cyst.

Postoperative Course. Postoperative MR images demonstrated a complete resection of the lesion (Fig. 3). The patient did well postoperatively with no neurological deficits. The remainder of the hospital course was uneventful.

\section{FAR-LATERAL TRANSCONDYLAR APPROACH}

\section{Preoperative Considerations}

The far-lateral transcondylar approach is performed on the side of the lateral extension of the lesion. If the lesion is strictly midline, the side of the approach can be chosen based on the anatomy of the VA, the sigmoid sinus, and the jugular bulbs. Al-Mefty, et al., ${ }^{2}$ have suggested approaching from the side of the nondominant VA or nondominant jugular bulb in these cases. Intraoperative monitoring, including somatosensory evoked potentials, auditory evoked respon- ses, facial nerve monitoring, and 10th, 11th, and 12th cranial nerve monitoring, should be implemented. The 10th cranial nerve is monitored with an electromyographic endotracheal tube, and the 11th and 12th nerves are monitored via electrodes placed directly into the sternocleidomastoid muscle and the tongue, respectively.

\section{Positioning and Skin Incision}

The patient is placed in the lateral position and the head is held in three-point pin fixation with the neck slightly flexed, the vertex angled slightly down, and the face rotated slightly ventrally, so that the ipsilateral external auditory meatus and the mastoid bone are at the highest point (Fig. 5). Positioning the head in this manner allows better exposure of the occipitocervical region and improves the inferior-to-superior viewing angle for the surgeon. An axillary roll is placed, and the patient's contralateral arm rests on a Krauss armrest. The elevated arm is distracted inferiorly toward the foot of the table to provide more room for the surgeon to maneuver above the shoulder. All pressure points are carefully padded with foam or gel pads. The patient is secured to the operating table with adhesive tape to 


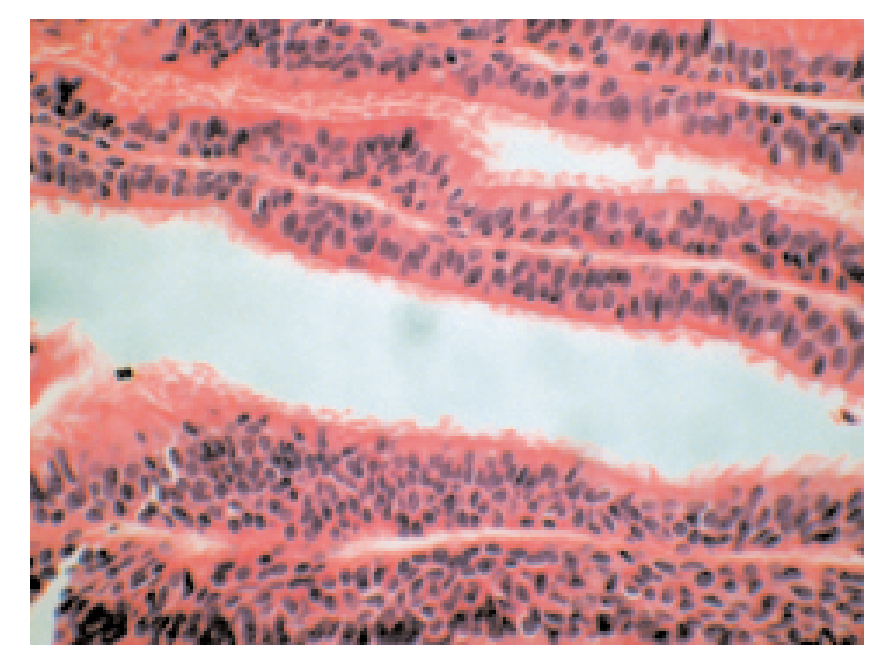

Fig. 4. Photomicrograph of the specimen obtained in Case 2, showing the cyst wall lined by ciliated simple cuboidal-to-columnar epithelium. $\mathrm{H} \& \mathrm{E}$, original magnification $\times 400$.

allow safe rotation of the table during the operation when needed to improve the surgeon's line of sight. Intravenous glucocorticoid agents, antibiotic drugs, and mannitol are administered at the time of the skin incision.

A retroauricular curvilinear skin incision starts approximately 2 to $3 \mathrm{~cm}$ behind the ear and continues inferiorly into the neck over the posterior border of the sternocleidomastoid muscle to C-3 or C-4 (Fig. 5). The skin flap is elevated in two layers: the incised skin and galea are first elevated to expose the underlying pericranium above the superficial neck fascia, which may be harvested as a fascial graft for later watertight dural closure. The pericranium and the superficial fascia are then elevated to expose the underlying musculature.

\section{Muscle Dissection and the Suboccipital Triangle}

Anatomically, three layers of muscle are identified during the dissection. The first, the superficial layer (trapezius and sternocleidomastoid), and the second, the middle layer (splenius capitis, longissimus capitis, and semispinalis capitis), are incised and reflected as a single layer to expose the suboccipital triangle (Fig. 6), which is bound by the third, deep layer of muscles (medially by the rectus capitis posterior major, inferiorly by the inferior oblique, and superolaterally by the superior oblique muscle). The rectus capitis major muscle inserts superiorly on the inferior nuchal line and inferiorly on the spinous process of C-2; the inferior oblique muscle inserts superiorly on the transverse process of C-1 and inferiorly on the spinous process of C-2; the superior oblique muscle inserts superiorly at the temporooccipital suture and inferiorly on the transverse process of $\mathrm{C}-1$. The suboccipital triangle, which involves the dorsal ramus of the $\mathrm{C}-1$ nerve root and the $\mathrm{V}_{3}$ (horizontal segment) of the VA, can be opened by detaching the insertions of the superior and inferior oblique muscles from the transverse process of $\mathrm{C}-1$ and reflecting them posteriorly. The rectus capitis major is detached from the inferior nuchal line and reflected posteriorly. The C-1 lamina and VA will then become more apparent. The VA is covered by a ve-

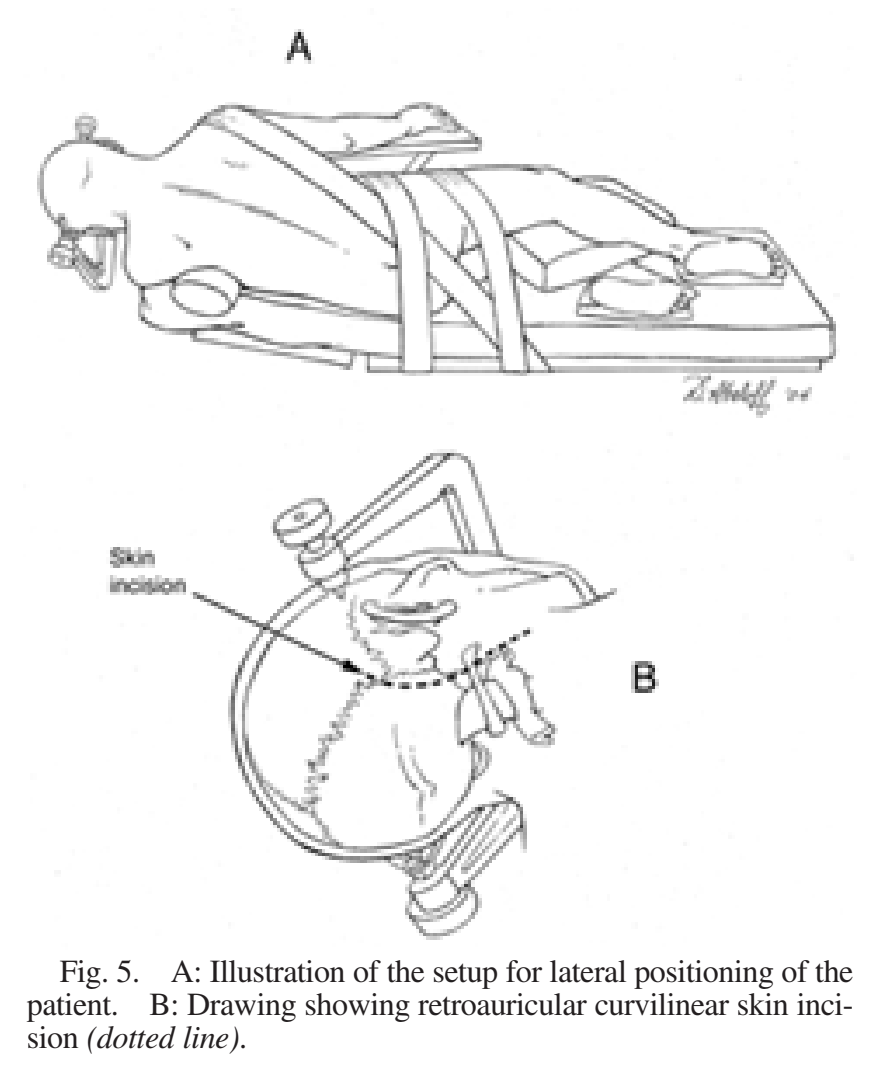

nous plexus, sometimes referred to as the suboccipital cavernous sinus. ${ }^{4}$ Further exposure of the laminae of C-2 or C-3 may be performed if more inferior exposure is needed.

\section{Exposure of the Extradural VA}

Exposure and control of the extradural VA is important and can be achieved by identifying its extradural course from the foramen transversarium of $\mathrm{C}-2$ to the occiput. The ventral ramus of the C-2 nerve root, which is found between the laminae of C-1 and C-2, can be traced laterally until it crosses dorsally to the vertical segment of the VA, coursing between the foramen transversarium of $\mathrm{C}-2$ and $\mathrm{C}-1$ (Fig. 6). ${ }^{2,15,23}$ As the VA exits the foramen transversarium of $\mathrm{C}-1$, it is encased in a venous plexus and courses posteriorly behind the lateral mass of $\mathrm{C}-1$ in the vertebral groove, then turns medially to pierce the atlantooccipital membrane and dura mater. Several small branches feeding the muscles, in addition to the posterior meningeal artery, arise from the horizontal segment of the VA, which can be safely coagulated. In some cases, the posterior spinal artery and posterior inferior cerebellar artery can arise extradurally and can potentially be injured. ${ }^{25,34}$ Subperiosteal dissection of the VA from the vertebral groove reduces bleeding from the venous plexus by leaving the periosteal sheath around the artery intact. ${ }^{19}$ The atlantooccipital membrane is sharply divided to expose the underlying dura mater.

\section{Suboccipital Craniectomy and C-1 Hemilaminectomy}

A lateral suboccipital craniectomy or craniotomy is initially performed with a high-speed drill and rongeurs. The 


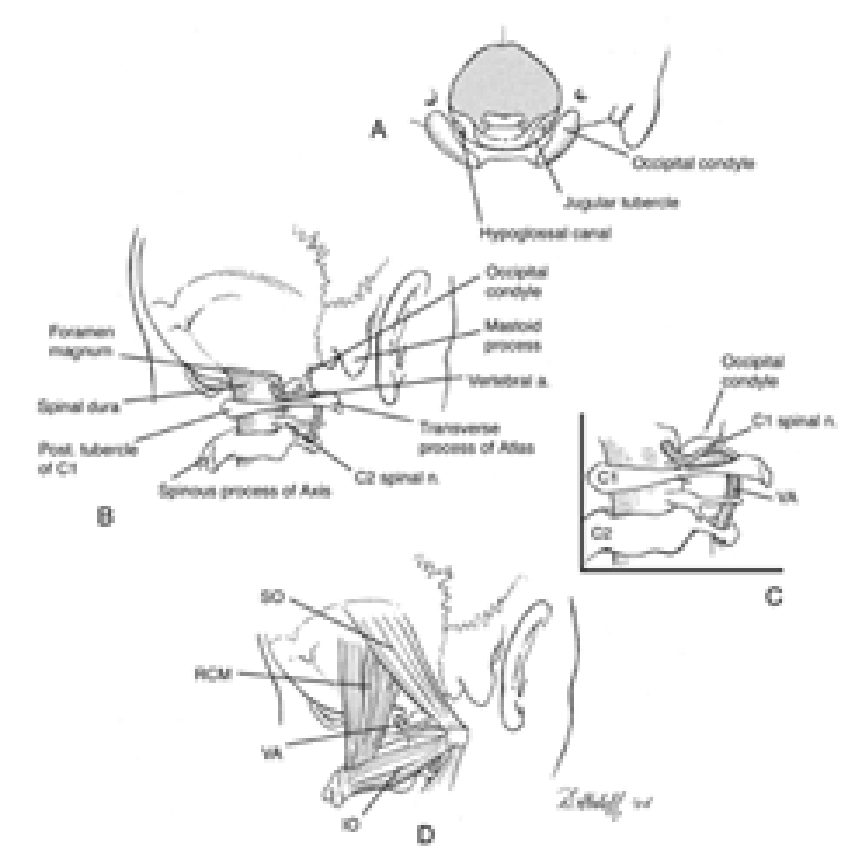

Fig. 6. Anatomical drawings. A: Inferior view of the base of the skull showing the anatomical relationship of the jugular tubercle, hypoglossal canal, occipital condyle, and foramen magnum. B: Surface anatomy and key landmarks. C: Course of the VA. Note that the ventral ramus of the $\mathrm{C}-2$ nerve root, found between the laminae of C-1 and C-2, can be traced laterally until it crosses dorsally to the vertical segment of the VA, coursing between the foramen transversarium of C-2 and C-1. D: The suboccipital triangle, which is bound medially by the rectus capitis posterior major (RCM) muscle, inferiorly by the inferior oblique (IO) muscle, and superolaterally by the superior oblique (SO) muscle, serves as an anatomical landmark for identifying the dorsal ramus of the C-1 nerve root and the $\mathrm{V}_{3}$ (horizontal segment) of the VA. A. = artery; n. $=$ nerve; post. $=$ posterior.

craniectomy usually extends toward the midline medially and to the inferior nuchal line superiorly (depending on the extent of exposure needed), to the posterior rim of the foramen magnum inferiorly, and up to the occipital condyle laterally (Fig. 7). To provide more superior access to the CPA, the craniectomy can be extended up to the transverse sigmoid junction. The sigmoid sinus and jugular bulb are exposed with rongeurs and a high-speed drill. The posterior condylar emissary vein will be encountered as it travels from the jugular bulb and exits the condylar fossa through the condylar canal to join the extradural venous plexus. Hemostasis can be achieved by packing the vessel with Surgicel. An ipsilateral hemilaminectomy of C-1 improves the dural exposure inferiorly. More inferior exposure for lower-lying lesions can be created by removing the hemilamina of C-2 and C-3, if desired.

\section{Transcondylar Resection}

Extradural reduction of the occipital condyle is one of the key maneuvers in maximizing exposure to the ventral aspect of the craniovertebral junction while avoiding brainstem retraction (Fig. 7). Anatomical morphometric studies have demonstrated that partial condylar resection increases the angle of exposure, the working space at the level of the foramen magnum, and the visualization of both the ventral and ventrolateral aspects of the craniovertebral junction and the contralateral aspect of the inferior clivus. ${ }^{11,31,33}$ The recommendations for the degree of occipital condyle removal vary greatly in the literature, ranging from no resection up to complete condyle removal. ${ }^{5,23,27-31}$ In our experience, removal of the posterior and medial third of the condyle is generally adequate if more ventral exposure is needed. If $50 \%$ or more of the condyle is resected or has been destroyed by the lesion, the instability of the craniovertebral junction increases and an occipitocervical stabilization should be strongly considered. ${ }^{32}$ Although extradural reduction of the jugular tubercle aids in maximizing intradural exposure across the anterior surface of the brainstem and mid-clivus, this maneuver is probably not necessary for neurenteric cysts because these lesions are soft and amenable to removal by suction.

\section{Intradural Exposure}

A curvilinear incision of the dura mater is made several millimeters posterior to the sigmoid sinus, extending inferiorly toward the C-2 lamina, staying posterior to the VA where it pierces the dura (Fig. 7). The dural opening may be extended anteriorly in a T-shaped fashion, and we prefer to do this just superior to the VA to attain greater exposure. A dural cuff is preserved around the VA for later watertight closure. The incision can be extended up to the junction of the transverse and sigmoid sinuses if more exposure of the CPA is needed. The anterior leaflet of dura mater is reflected laterally and held with tacking sutures for maximal exposure. Adequate reduction of the occipital condyle should provide a straight surgical trajectory to the

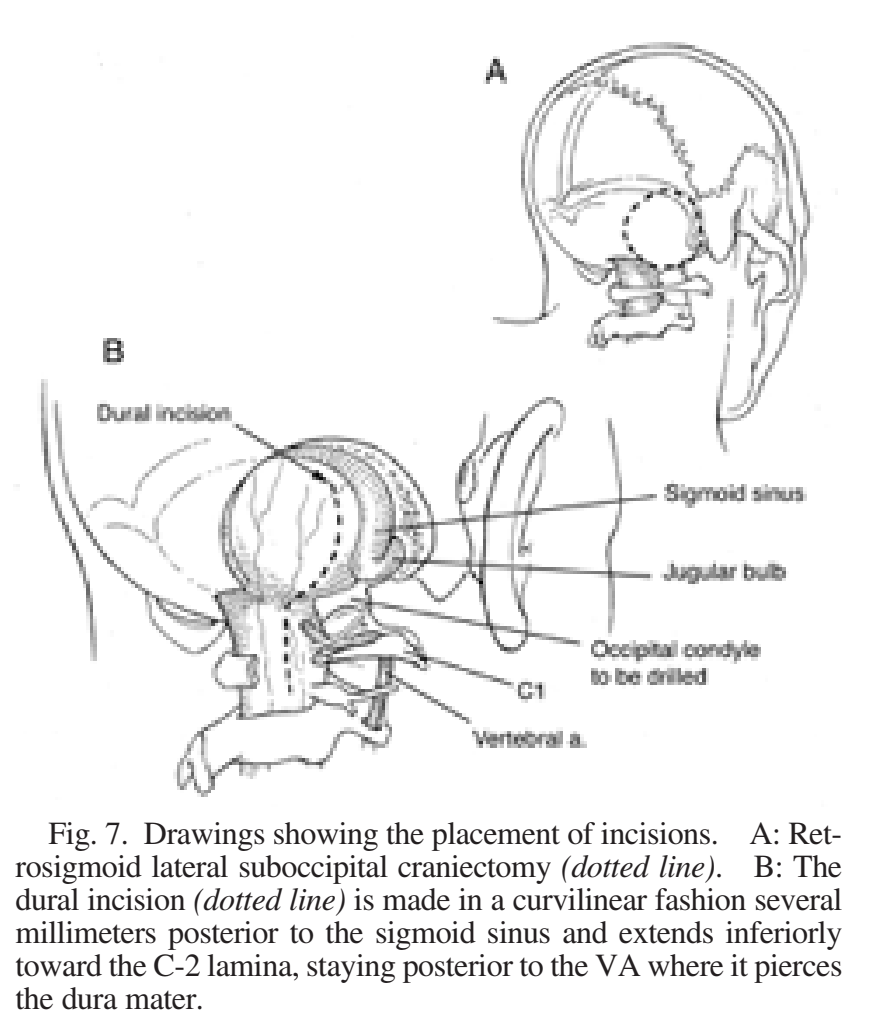


craniovertebral junction parallel to the intracranial course of the VA. ${ }^{9}$ Structures of the inferior aspect of the CPA and the cerebellomedullary angle are visualized. Sharp arachnoid dissection is performed and the following structures can be visualized: the fifth through 12th cranial nerves; the basilar artery; the VA; the vertebrobasilar junction; the posterior inferior cerebellar artery; and the anterior inferior cerebellar artery (Fig. 8). Complete resection of the cyst wall and contents is then performed, with strict adherence to microsurgical technique and preservation of all cranial and spinal nerves and vascular structures.

\section{Wound Closure}

A primary watertight closure of the dura mater should be performed. If necessary, an autologous pericranium or fascial graft can be harvested from the neck wound. This can be supplemented with autologous fat and fibrin glue. The exposed mastoid air cells are closed with bone wax. The muscle layers are carefully reapproximated to avoid postoperative cerebrospinal fluid leakage. Temporary cerebrospinal fluid diversion with a lumbar drain can promote sealing of the wound and reduce the risk that a pseudomeningocele will develop.

\section{DISCUSSION}

\section{Clinical Presentation}

These lesions are frequently incidental neuroimaging findings, but occasionally they may produce symptoms of progressive neurological impairment and chronic increased intracranial pressure. ${ }^{8}$ Neurenteric cysts of the cervicomedullary junction can cause symptoms by an inflammatory reaction or by mass effect. Active secretion by goblet cells can result in cyst expansion and compression of neighboring neurovascular structures. ${ }^{14}$ The most frequent complaint is headaches, followed by gait disturbance and motor and sensory disturbances. ${ }^{6}$ Intermittent leakage of cyst contents

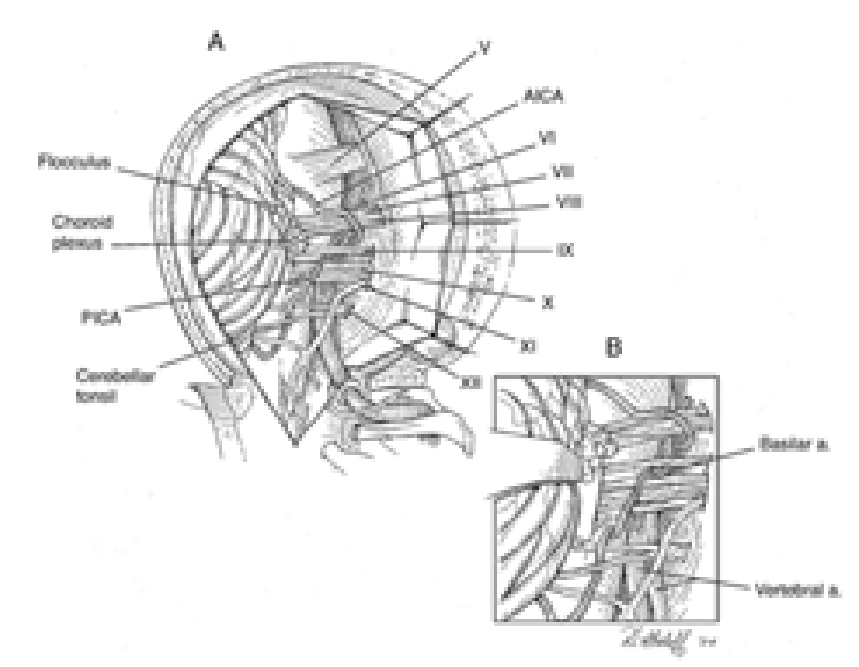

Fig. 8. Diagrams illustrating the intradural exposure of the farlateral transcondylar approach. AICA = anterior inferior cerebellar artery; PICA = posterior inferior cerebellar artery; V-XII = fifth through 12 th cranial nerves. can result in recurrent aseptic meningitis. ${ }^{26}$ Involvement of the cranial nerves can result in facial numbness, hemifacial spasm, hearing loss, and swallowing difficulties.

\section{Neuroimaging Characteristics}

The imaging characteristics of neurenteric cysts can be quite variable. This may be attributed to the presence of proteinaceous or other hydrophilic contents. ${ }^{8}$ Computerized tomography scanning usually reveals a hypodense lesion in most cases, although occasionally the lesions can be hyperdense. Sometimes the cyst may be undetectable on computerized tomography scans, and MR imaging is therefore the study of choice for evaluating neurenteric cysts. These extramedullary cystic lesions may appear hypointense, isointense, or slightly hyperintense on $\mathrm{T}_{1}$-weighted MR images and mostly hyperintense on $\mathrm{T}_{2}$-weighted and FLAIR images without edema. The cyst wall does not enhance after Gd injection in most cases. ${ }^{26}$ In the patient presented in Case 2, however, the cyst enhanced after Gd administration. Unlike epidermoid cysts, neurenteric cysts do not restrict on diffusion-weighted images.

\section{Histopathological Findings}

Intraoperatively, neurenteric cysts appear as yellow or milky-white lesions filled with gelatinous or mucoid fluid. Histologically, they are benign lesions characterized by a cyst wall lined with simple or pseudostratified cuboidal-tocolumnar epithelium with a basement membrane resembling those of the respiratory and intestinal tracts. ${ }^{18}$ The epithelium is sometimes ciliated and often contains mucinsecreting goblet cells that exhibit PAS-positive staining. The intracystic fluid is usually milky, xanthochromic, or transparent. ${ }^{14}$ Immunohistochemical studies are often useful in the diagnosis. Neurenteric cysts usually exhibit positive staining for keratin, carcinoembryonic antigen, and epithelial membrane antigen. They are negative for glial fibrillary acidic protein, neuron-specific enolase, $\mathrm{S} 100$, and vimentin staining. This pattern of immunoreactivity confirms an endodermal origin of neurenteric cysts. ${ }^{6,18,26}$

\section{Surgical Management}

Because neurenteric cysts are benign lesions, the goal of surgery should be complete excision of both the cyst contents and the cyst wall if the dissection can be performed without injury to the neighboring neurovascular structures. ${ }^{6,8,14}$ If the cyst wall appears strongly adherent to the brainstem or vasculature, subtotal resection is a reasonable alternative to avoid injury to these structures. In these cases, patients should be carefully monitored with serial MR images obtained to detect cyst recurrence. Neurenteric cysts of the cervicomedullary junction often require a skull base approach to gain access to the lesion. We prefer the far-lateral transcondylar approach because it allows access to ventrally and ventrolaterally located lesions of the cervicomedullary junction. Access to the entire CPA up to the tentorium can be achieved, if needed.

\section{Acknowledgments}

We thank Diane Abeloff for her excellent drawings of the anatomy and Kristin Kraus for her editorial assistance. 


\section{References}

1. Abe K, Oyama K, Mori K, et al: Neurenteric cyst of the craniocervical junction - case report. Neurol Med Chir 39:875-880, 1999

2. al-Mefty O, Borba LA, Aoki N, et al: The transcondylar approach to extradural nonneoplastic lesions of the craniovertebral junction. J Neurosurg 84:1-6, 1996

3. Arai Y, Yamauchi Y, Tsuji T, et al: Spinal neurenteric cyst. Report of two cases and review of forty-one cases reported in Japan. Spine 17:1421-1424, 1992

4. Arnautovic KI, al-Mefty O, Pait TG, et al: The suboccipital cavernous sinus. J Neurosurg 86:252-262, 1997

5. Babu RP, Sekhar LN, Wright DC: Extreme lateral transcondylar approach: technical improvements and lessons learned. J Neurosurg 81:49-59, 1994

6. Bejjani GK, Wright DC, Schessel D, et al: Endodermal cysts of the posterior fossa. Report of three cases and review of the literature. J Neurosurg 89:326-335, 1998

7. Birch BD, McCormick PC: High cervical split cord malformation and neurenteric cyst associated with congenital mirror movements: case report. Neurosurgery 38:813-816, 1996

8. Cheng JS, Cusick JF, Ho KC, et al: Lateral supratentorial endodermal cyst: case report and review of literature. Neurosurgery 51:493-499, 2002

9. Day JD, Fukushima T, Giannotta SL: Cranial base approaches to posterior circulation aneurysms. J Neurosurg 87:544-554, 1997

10. Devkota UP, Lam JM, Ng H, et al: An anterior intradural neurenteric cyst of the cervical spine: complete excision through central corpectomy approach-case report. Neurosurgery 35: 1150-1154, 1994

11. Dowd GC, Zeiller S, Awasthi D: Far lateral transcondylar approach: dimensional anatomy. Neurosurgery 45:95-100, 1999

12. Ergun R, Akdemir G, Gezici AR, et al: Craniocervical neurenteric cyst without associated abnormalities. Pediatr Neurosurg 32:95-99, 2000

13. Eynon-Lewis NJ, Kitchen N, Scaravilli F, et al: Neurenteric cyst of the cerebellopontine angle: case report. Neurosurgery 42:655-658, 1998

14. Filho FL, Tatagiba M, Carvalho GA, et al: Neurenteric cyst of the craniocervical junction. Report of three cases. J Neurosurg 94:129-132, 2001

15. Fukushima T (ed): Manual of Skull Base Dissection. Pittsburgh: AF Neuro Video, 1996, pp 111-123

16. Fuse T, Yamada K, Kamiya K, et al: Neurenteric cyst at the craniovertebral junction: report of two cases. Surg Neurol 50: 431-436, 1998

17. Graziani N, Dufour H, Figarella-Branger D, et al: Do the suprasellar neurenteric cyst, the Rathke cleft cyst and the colloid cyst constitute a same entity? Acta Neurochir 133:174-180, 1995

18. Harris CP, Dias MS, Brockmeyer DL, et al: Neurenteric cysts of the posterior fossa: recognition, management, and embryogenesis. Neurosurgery 29:893-898, 1991

19. Kawashima M, Tanriover N, Rhoton AL Jr, et al: Comparison of the far lateral and extreme lateral variants of the atlanto-occipital transarticular approach to anterior extradural lesions of the craniovertebral junction. Neurosurgery 53:662-675, 2003
20. Lazareff JA, Hoil Parra JA: Intradural neurenteric cyst at the craniovertebral junction. Childs Nerv Syst 11:536-538, 1995

21. Lee WY, Tseng HM, Lin MC, et al: Neurenteric cyst at craniocervical junction: report of a case. J Formos Med Assoc 91: 722-724, 1992

22. Mann KS, Khosla VK, Gulati DR, et al: Spinal neurenteric cyst. Association with vertebral anomalies, diastematomyelia, dorsal fistula, and lipoma. Surg Neurol 21:358-362, 1984

23. Nanda A, Vincent DA, Vannemreddy PS, et al: Far-lateral approach to intradural lesions of the foramen magnum without resection of the occipital condyle. J Neurosurg 96:302-309, 2002

24. Prasad VS, Reddy DR, Murty JM: Cervico-thoracic neurenteric cyst: clinicoradiological correlation with embryogenesis. Childs Nerv Syst 12:48-51, 1996

25. Rhoton AL Jr: The far-lateral approach and its transcondylar, supracondylar, and paracondylar extensions. Neurosurgery 47 (Suppl 1):S195-S209, 2000

26. Sahara Y, Nagasaka T, Takayasu M, et al: Recurrence of a neurenteric cyst with malignant transformation in the foramen magnum after total resection. Case report. J Neurosurg 95: 341-345, 2001

27. Salas E, Sekhar LN, Ziyal IM, et al: Variations of the extremelateral craniocervical approach: anatomical study and clinical analysis of 69 patients. J Neurosurg 90:206-219, 1999

28. Samii M, Klekamp J, Carvalho G: Surgical results for meningiomas of the craniocervical junction. Neurosurgery 39: 1086-1095, 1996

29. Sen C: The transcondylar approach to the lower clivus, foramen magnum and C1-C2. Clin Neurosurg 43:113-126, 1996

30. Sen CN, Sekhar LN: An extreme lateral approach to intradural lesions of the cervical spine and foramen magnum. Neurosurgery 27:197-204, 1990

31. Spektor S, Anderson GJ, McMenomey SO, et al: Quantitative description of the far-lateral transcondylar transtubercular approach to the foramen magnum and clivus. J Neurosurg 92: 824-831, 2000

32. Vishteh AG, Crawford NR, Melton MS, et al: Stability of the craniovertebral junction after unilateral occipital condyle resection: a biomechanical study. J Neurosurg 90:91-98, 1999

33. Wanebo JE, Chicoine MR: Quantitative analysis of the transcondylar approach to the foramen magnum. Neurosurgery 49: 934-943, 2001

34. Wen HT, Rhoton AL Jr, Katsuta T, et al: Microsurgical anatomy of the transcondylar, supracondylar, and paracondylar extensions of the far-lateral approach. J Neurosurg 87:555-585, 1997

Manuscript received June 20, 2005.

Accepted in final form July 15, 2005.

Address reprint requests to: William T. Couldwell, M.D., Ph.D., Department of Neurosurgery, University of Utah School of Medicine, 30 North 1900 East, Suite 3B409, Salt Lake City, Utah 84132. email: william.couldwell@hsc.utah.edu. 